\title{
THE PROGRESS OF A SYNTACTIC CHANGE ENGLISH DO-SUPPORT*
}

1.1. The English construction with do-support in the affirmative form, in the interrogative form, in the declarative-negative form, and in the interrogative-negative form is a periphrastic construction. It satisfied the definition of a strengthened construction as long as it was an optional replacement of the corresponding non-strengthened construction, i.e. of forms without do-support.

1.2. The following predictions can be made about the use of the construction with do-support while it was a strengthened construction:

1.2.1. The construction with do-support was used more often than the construction without $d o$-support with heavy main verbs, i.e. within frequent and with morphologically complex verbs.

1.2.2. The construction with $d o$-support was used relatively more frequently than the construction without $d o$-support under complex grammatical conditions:

a) in dependent clauses (vis-à-vis main clauses)

b) in the non-present tenses (vis-à-vis the present tense)

c) in the non-indicative moods (vis-à-vis the indicative mood)

d) in the non-third verbal persons (vis-à-vis the third verbal person)

e) in the non-singular (vis-à-vis the singular)

f) in non-declarative clauses (vis-à-vis declarative clauses)

g) in non-affirmative declarative clauses (vis-à-vis affirmative declarative clauses)

h) with verb + prepositional object (vis-à-vis verb + direct object)

i) with verb + object clause (vis-à-vis verb + direct object).

\section{THE CONSTRUCTION WITH DO-SUPPORT IN THE CORPUS}

2.1. The corpus consists of John Lyly's plays in prose (Campaspe $1580 / \mathrm{C} /$, Sapho and Phao 1581 /SP/, Gallathea 1584 /G/, Endimion 1585 /E/, Midas 1589

\footnotetext{
* This article is an abstract of the author's M.A. thesis, "The Development of a Syntactic Change: The English Verb 'Do'“, defended at the Faculty of Philosophy in Ljubljana on June 21, 1988 and was part of the requirements for his M.A. degree. Advisor: Prof. Dr. Janez Orešnik.
} 
/M/, Mother Bombie 1590 /MB/, Loves Metamorphosis 1599 /LM/ as edited by Bond 1902.

2.2. The examples of the construction with do-support in the corpus will now be adduced, grouped according to the propositional modality of the construction (affirmative, negative, interrogative, and interrogative-negative), and subclassified as indicated in paragraph 1.2.2. The interrogative form is classified into yes-no questions and wh-questions.

\subsection{The affirmative form}

2.3.1. $\mathrm{C}=\mathrm{m}, \mathrm{T}=\mathrm{pr}, \mathrm{N}=\mathrm{sg}, \mathrm{P}=1, \mathrm{~V}=\operatorname{tr}, \mathrm{O}=\operatorname{dir} . \mathrm{obj}^{1}$

1. Thy name I doo remember $-\mathrm{E}$ V.1.54. ${ }^{2}$

2. Here I do plight my faith - MB IV.1.47.

3. And I doo maintaine Accius - MB V.3.345.

2.3.2. $\mathrm{C}=\mathrm{m}, \mathrm{T}=\mathrm{pr}, \mathrm{N}=\mathrm{sg}, \mathrm{P}=1, \mathrm{~V}=\mathrm{tr}, \mathrm{O}=\operatorname{dir} . \mathrm{obj}, \mathrm{i}$

4. here doe I beare them in my handes. - SP I.3.5.

5. What a life doe I leade - G II.3.9.

6. for now only do I thinke Eumenides faithfull - E V.3.234.

7. so doo I thinke it most allowable - M III.3.65.

8. yet doe I find a kinde of sweetnesse. - LM III.1.136.

9. for as great sport doe I take - LM I.2.24.

2.3.3. $\mathrm{C}=\mathrm{m}, \mathrm{T}=\mathrm{pr}, \mathrm{N}=\mathrm{sg}, \mathrm{P}=1, \mathrm{~V}=\operatorname{tr}, \mathrm{O}=$ dir.obj, Prn $=$ rfl.prn, $\mathrm{i}$ 10. and therefore doe I esteeme my selfe most free - E III.2.7.

2.3.4. $\mathrm{C}=\mathrm{m}, \mathrm{T}=\mathrm{pr}, \mathrm{N}=\mathrm{sg}, \mathrm{P}=1, \mathrm{~V}=\mathrm{tr}, \mathrm{O}=$ obj.cl, $\mathrm{i}$

11. And rather doe I wish hee preserue our fame - C I.1.55.

12. els do I think there is none in their harts. - SP III.4.30.

13. Yet doe I rather choose to haue my tongue cut out $-\mathrm{E}$ V.3.216.

14. so do I meane to do againe - C II.1.43.

15. now onely doe I begin to liue. - E V.3.214.

2.3.5. $\mathrm{C}=\mathrm{m}, \mathrm{T}=\mathrm{pr}, \mathrm{N}=\mathrm{sg}, \mathrm{P}=1, \mathrm{~V}=\mathrm{tr}, \mathrm{O}=$ prep.obj

16. I doe agree thereto $-\mathrm{G}$ I.1.10.

2.3.6. $\mathrm{C}=\mathrm{m}, \mathrm{T}=\mathrm{pr}, \mathrm{N}=\mathrm{sg}, \mathrm{P}=2, \mathrm{~V}=\operatorname{tr}, \mathrm{O}=\operatorname{dir} . \mathrm{obj}$

17. thou doost decrease thy glemes - E I.1.63.

18. thou dost want wit - M I.2.55.

2.3.7. $\mathrm{C}=\mathrm{m}, \mathrm{T}=\mathrm{pr}, \mathrm{N}=\mathrm{sg}, \mathrm{P}=2, \mathrm{~V}=\operatorname{tr}, \mathrm{O}=\operatorname{dir} . \mathrm{obj}, \mathrm{i}$

19. What perplexities dost thou feele! - SP IV.2.7.

20. little doest thou know thy hard fortune - MB II.3.99. 
2.3.8. $\mathrm{C}=\mathrm{m}, \mathrm{T}=\mathrm{pr}, \mathrm{N}=\mathrm{sg}, \mathrm{P}=2, \mathrm{~V}=\operatorname{tr}, \mathrm{O}=$ obj.cl, $\mathrm{i}$ 21. Little doost thou knowe Endimion when thou shalt wake - E II.3.24. 22. for little doost thou knowe heauie his head lies - E IV.1.73.

2.3.9. $\mathrm{C}=\mathrm{m}, \mathrm{T}=\mathrm{pr}, \mathrm{N}=\mathrm{sg}, \mathrm{P}=2, \mathrm{~V}=\mathrm{tr}, \mathrm{O}=$ prep.obj 23. But thou ...dost detract from thy perfections - E I.1.60.

2.3.10. $\mathrm{C}=\mathrm{m}, \mathrm{T}=\mathrm{pr}, \mathrm{N}=\mathrm{sg}, \mathrm{P}=3, \mathrm{~V}=\operatorname{tr}, \mathrm{O}=\operatorname{dir} . \mathrm{obj}$ 24. Semele dooth possesse my loue. - E III.4.116.

2.3.11. $\mathrm{C}=\mathrm{m}, \mathrm{T}=\mathrm{pr}, \mathrm{N}=\mathrm{sg}, \mathrm{P}=3, \mathrm{~V}=\mathrm{tr}, \mathrm{O}=\operatorname{dir} . o b j, \mathrm{i}$ 25. Then doth he shew me coũterfeits - C I.2.67.

26. As much doth it delight thee - SP I.1.7.

2.3.12. $\mathrm{C}=\mathrm{m}, \mathrm{T}=\mathrm{pr}, \mathrm{N}=\mathrm{sg}, \mathrm{P}=3, \mathrm{~V}=\mathrm{tr}, \mathrm{O}=$ obj.cl 27. Aristotle doth denie. - C I.3.74.

2.3.13. $\mathrm{C}=\mathrm{m}, \mathrm{T}=\mathrm{pr}, \mathrm{N}=\mathrm{sg}, \mathrm{P}=3, \mathrm{~V}=\mathrm{tr}, \mathrm{O}=$ prep.obj 28. The Sunne dooth beate vppon the playne fieldes $-\mathrm{G}$ I.1.1.

29. my hart doth nowe take hold of me. - E III. 4.37.

2.3.14. $\mathrm{C}=\mathrm{m}, \mathrm{T}=\mathrm{pr}, \mathrm{N}=\mathrm{sg}, \mathrm{P}=3, \mathrm{~V}=$ intr

30. for Alexander doth loue - C II.2.96.

31. my maister doth come. - C III.5.3.

32. thy tongue doth itch - M II.1.116.

33. Thy father doth liue - MB III.4.162.

2.3.15. $\mathrm{C}=\mathrm{m}, \mathrm{T}=\mathrm{pr}, \mathrm{N}=\mathrm{sg}, \mathrm{P}=3, \mathrm{~V}=$ intr, $\mathrm{i}$ 34. and often doth it happen - G III.3.24.

35. so doth my wit increase by loue. - E III.3.62.

2.3.16. $\mathrm{C}=\mathrm{m}, \mathrm{T}=\mathrm{pr}, \mathrm{N}=\mathrm{pl}, \mathrm{P}=2, \mathrm{~V}=\operatorname{tr}, \mathrm{O}=\operatorname{dir} . \mathrm{obj}, \mathrm{i}$ 36. Litle do you know - C II.2.79.

37. and therefore sleightly do you regarde - C II.2.79.

$$
\text { 2.3.17. } \mathrm{C}=\mathrm{m}, \mathrm{T}=\mathrm{pr}, \mathrm{N}=\mathrm{pl}, \mathrm{P}=3, \mathrm{~V}=\mathrm{tr}, \mathrm{O}=\text { obj.cl }
$$

38. Gods do know,..., that loue is a consuming of wit - LM III.1.23.

$$
\text { 2.3.18. } \mathrm{C}=\mathrm{m}, \mathrm{T}=\mathrm{pr}, \mathrm{N}=\mathrm{pl}, \mathrm{P}=3, \mathrm{~V}=\operatorname{intr}
$$

39. sing a song to the tune of my teeth do ake. - M III.2.136.

$$
\text { 2.3.19. } \mathrm{C}=\mathrm{m}, \mathrm{T}=\mathrm{pr}, \mathrm{N}=\mathrm{pl}, \mathrm{P}=3, \mathrm{~V}=\text { intr, } \mathrm{i}
$$

40. nowe do they dine vpon another. - C II.1.52.

41. So do knaues go double - MB IV.1.9. 
2.3.20. $\mathrm{C}=\mathrm{m}, \mathrm{T}=\mathrm{pa}, \mathrm{N}=\mathrm{sg}, \mathrm{P}=1, \mathrm{~V}=\mathrm{tr}, \mathrm{O}=\operatorname{dir} . \mathrm{obj}, \mathrm{i}$ 42. there did I weare Pantopheles - SP I.3.4.

43. in the rose did I always behold thy colour - LM V.4.121.

2.3.21. $\mathrm{C}=\mathrm{m}, \mathrm{T}=\mathrm{pa}, \mathrm{N}=\mathrm{sg}, \mathrm{P}=1, \mathrm{~V}=\mathrm{tr}, \mathrm{O}=$ obj.cl 44. I did euer imagine that trou loue would end - LM V.3.3.

2.3.22. $\mathrm{C}=\mathrm{m}, \mathrm{T}=$ pa, $\mathrm{N}=\mathrm{sg}, \mathrm{P}=1, \mathrm{~V}=\mathrm{tr}, \mathrm{O}=$ obj.cl, $\mathrm{i}$ 45. A bridge of gold did I mean to make - M III.1.46.

46. Those Ilandes did I long to touch - M III.1.48.

47. Vpon that Rocke did I resolue to end my life: - LM V.4.116.

2.3.23. $C=m, T=p a, N=s g, P=2, V=\operatorname{tr}, O=\operatorname{dir}$.obj 48. thou didst forget thy dutie - C I.3.111.

2.3.24. $\mathrm{C}=\mathrm{m}, \mathrm{T}=$ pa, $\mathrm{N}=\mathrm{sg}, \mathrm{P}=2, \mathrm{~V}=\mathrm{tr}, \mathrm{O}=$ obj.cl, $\mathrm{i}$ 49. Thys noble Gentleman Geron...didst thou procure to lyue in a Deserte E V.3.35.

2.3.25. $\mathrm{C}=\mathrm{m}, \mathrm{T}=\mathrm{pa}, \mathrm{N}=\mathrm{sg}, \mathrm{P}=3, \mathrm{~V}=\operatorname{tr}, \mathrm{O}=\operatorname{dir} . \mathrm{obj}$ 50. But Fortune...did change her copie - G I.1.20.

51. Erisicthon did...hewe downe my sacred tree. - LM V.1.32.

2.3.26. $\mathrm{C}=\mathrm{m}, \mathrm{T}=\mathrm{pa}, \mathrm{N}=\mathrm{sg}, \mathrm{P}=3, \mathrm{~V}=\mathrm{tr}, \mathrm{O}=\operatorname{dir} . \mathrm{obj}, \mathrm{i}$ 52. and the same time did Mars make a full point - SP III.2.49.

53. That shower did my Master make - G II.3.39.

54. So did Iupiter...possesse his loue - M II.1.13.

2.3.27. $\mathrm{C}=\mathrm{m}, \mathrm{T}=\mathrm{pa}, \mathrm{N}=\mathrm{pl}, \mathrm{P}=2, \mathrm{~V}=\operatorname{tr}, \mathrm{O}=\operatorname{dir} . \mathrm{obj}, \mathrm{i}$ 55. so did you ouer-heare me - M III.2.75.

2.3.28 $\mathrm{C}=\mathrm{m}, \mathrm{T}=$ pa, $\mathrm{N}=$ pl, $\mathrm{P}=3, \mathrm{~V}=\operatorname{tr}, \mathrm{O}=\operatorname{dir} . \mathrm{obj}$ 56. Thy Nymphes did...scorne my constant love. - LM V.1.33.

2.3.29. $\mathrm{C}=\mathrm{d}, \mathrm{T}=\mathrm{pr}, \mathrm{N}=\mathrm{sg}, \mathrm{P}=1, \mathrm{~V}=$ intr 57. lest I doe starue - LM III.2.57.

2.3.30. $\mathrm{C}=\mathrm{d}, \mathrm{T}=\mathrm{pr}, \mathrm{N}=\mathrm{sg}, \mathrm{P}=3, \mathrm{~V}=\operatorname{tr}, \mathrm{O}=$ dir.obj 58. When thy mistresse doth bend her brow - SP II.4.107.

59. which Ioue doth worship - LM IV.1.60.

60. This is all Cupid doth commaund. - LM IV.1.123.

2.3.31. $\mathrm{C}=\mathrm{d}, \mathrm{T}=\mathrm{pr}, \mathrm{N}=\mathrm{sg}, \mathrm{P}=3, \mathrm{~V}=\mathrm{tr}, \mathrm{O}=\operatorname{dir} . \mathrm{obj}, \mathrm{i}$ 61. that dooth Nature claime. - M III.1.56. 
2.3.32. $\mathrm{C}=\mathrm{d}, \mathrm{T}=\mathrm{pr}, \mathrm{N}=\mathrm{sg}, \mathrm{P}=3, \mathrm{~V}=\mathrm{tr}, \mathrm{O}=$ obj.cl

62. which your learning doth well vnderstande is all man $-\mathrm{E}$ I.3.99.

2.3.33. $\mathrm{C}=\mathrm{d}, \mathrm{T}=\mathrm{pr}, \mathrm{N}=\mathrm{sg}, \mathrm{P}=3, \mathrm{~V}=$ intr

63. And whilst our flocke doth roame $-\mathrm{G}$ I.1.6.

64. then commonly nature dooth afforde - E V.3.20.

65. when a Lion doeth so much degenerat - M IV.2.25.

66. because he doth die - MB III.4.162.

2.3.34. $\mathrm{C}=\mathrm{d}, \mathrm{T}=\mathrm{pr}, \mathrm{N}=\mathrm{pl}, \mathrm{P}=3, \mathrm{~V}=\operatorname{tr}, \mathrm{O}=\operatorname{dir} .0 \mathrm{bj}$

67. whose passions and thoughts do...exceede others - C II.2.81.

68. that women do so lightly beleeue. - SP II.4.74.

69. where fowles doe breede theyr quils: - G I.1.31.

2.3.35. $\mathrm{C}=\mathrm{d}, \mathrm{T}=\mathrm{pr}, \mathrm{N}=\mathrm{pl}, \mathrm{P}=3, \mathrm{~V}=\operatorname{tr}, \mathrm{O}=\operatorname{dir} . \mathrm{obj}, \mathrm{i}$ 70. that doo the Gods chalenge. - M III.1.55.

2.3.36. $\mathrm{C}=\mathrm{d}, \mathrm{T}=\mathrm{pr}, \mathrm{N}=\mathrm{pl}, \mathrm{P}=3, \mathrm{~V}=\mathrm{tr}, \mathrm{O}=$ intr

71. whom other mens hornes do make to beware. - M V.2.39.

2.3.37. $\mathrm{C}=\mathrm{d}, \mathrm{T}=\mathrm{pa}, \mathrm{N}=\mathrm{sg}, \mathrm{P}=1, \mathrm{~V}=\operatorname{tr}, \mathrm{O}=\operatorname{dir} . \mathrm{obj}$ 72. I did quip thee $-\mathrm{C}$ III.2.28.

73. I did flatter thee - G IV.4.11.

74. I that did possesse mynes of golde - M III.1.7.

2.3.38. $\mathrm{C}=\mathrm{d}, \mathrm{T}=\mathrm{pa}, \mathrm{N}=\mathrm{sg}, \mathrm{P}=1, \mathrm{~V}=\mathrm{tr}, \mathrm{O}=$ obj.cl 75. I didde meane to make my tale. - SP I.1.68.

2.3.39. $\mathrm{C}=\mathrm{d}, \mathrm{T}=$ pa, $\mathrm{N}=\mathrm{sg}, \mathrm{P}=1, \mathrm{~V}=$ intr

76. as I did lye - SP V.3.20.

2.3.40. $\mathrm{C}=\mathrm{d}, \mathrm{T}=\mathrm{pa}, \mathrm{N}=\mathrm{sg}, \mathrm{P}=2, \mathrm{~V}=\operatorname{tr}, \mathrm{O}=$ dir.obj-

77. that thou didst counterfeate monye. - C I.3.121.

78. thou didst hit my hearte - SP IV.1.11.

79. thou didst both dislike and dishonour; - M IV.1.163.

80. which thou didst promise - LM III.2.25.

81. thou...that did/st/ sweare to follow. LM IV.2.87.

2.3.41. $\mathrm{C}=\mathrm{d}, \mathrm{T}=\mathrm{pa}, \mathrm{N}=\mathrm{sg}, \mathrm{P}=2, \mathrm{~V}=\operatorname{tr}, \mathrm{O}=$ dir.obj, Prn $=$ rfl.prn 82. thou didst vowe thy selfe to death $-\mathrm{E}$ V.3.159.

2.3.42. $\mathrm{C}=\mathrm{d}, \mathrm{T}=$ pa, $\mathrm{N}=\mathrm{sg}, \mathrm{P}=2, \mathrm{~V}=\operatorname{tr}, \mathrm{O}=\operatorname{dir}$.obj, $\mathrm{M}=$ cond, $\mathrm{i}$ 83. And didst thou want al things thou desirest - SP II.1.9. 
2.3.43. $\mathrm{C}=\mathrm{d}, \mathrm{T}=$ pa, $\mathrm{N}=\mathrm{sg}, \mathrm{P}=2, \mathrm{~V}=\mathrm{tr}, \mathrm{O}=$ prep.obj

84. onelie because thou didst shine vpon them - E II.1.19.

2.3.44. $\mathrm{C}=\mathrm{d}, \mathrm{T}=\mathrm{pa}, \mathrm{N}=\mathrm{sg}, \mathrm{P}=2, \mathrm{~V}=$ intr

85. when thy selfe didst loue - LM III.2.26.

2.3.45. $\mathrm{C}=\mathrm{d}, \mathrm{T}=\mathrm{pa}, \mathrm{N}=\mathrm{sg}, \mathrm{P}=3, \mathrm{~V}=\mathrm{tr}, \mathrm{O}=$ dir.obj

86. he did spring the partridge. - M IV.3.53.

2.3.46. $\mathrm{C}=\mathrm{d}, \mathrm{T}=\mathrm{pa}, \mathrm{N}=\mathrm{pl}, \mathrm{P}=3, \mathrm{~V}=\operatorname{tr}, \mathrm{O}=$ dir.obj, Prn $=$ rfl. prn 87. many wolues...did...bleede themselues to death - E V.1.121.

2.3.47. $\mathrm{C}=\mathrm{d}, \mathrm{T}=\mathrm{pa}, \mathrm{N}=$ you1, $\mathrm{P}=2, \mathrm{~V}=$ intr

88. you did seeme - LM V.4.127.

\subsection{The negative-declarative form}

2.4.1. $\mathrm{C}=\mathrm{m}, \mathrm{T}=\mathrm{pr}, \mathrm{N}=\mathrm{sg}, \mathrm{P}=1, \mathrm{~V}=\operatorname{tr}, \mathrm{O}=$ dir.obj

89. I doe not thinke it - G III.2.34.

90. thy fauour I doe not yet call - E V.1.55.

2.4.2. $\mathrm{C}=\mathrm{m}, \mathrm{T}=\mathrm{pr}, \mathrm{N}=\mathrm{sg}, \mathrm{P}=1, \mathrm{~V}=\mathrm{tr}, \mathrm{O}=$ obj.cl

91. I doe not think but his excuse wilbe better - C I.3.55.

92. I do not think he will flye. - C III.2.58.

93. I doe not wish thee to be a woman - G III.2.9.

94. I do not thinke that this breast shalbe pestred - E II.2.136.

95. I doe not thinke Loue hath any sparke - LM I.1.9.

96. I doe not disdaine that which I thinke is not - LM III.1.12.

2.4.3. $\mathrm{C}=\mathrm{m}, \mathrm{T}=\mathrm{pr}, \mathrm{N}=\mathrm{sg}, \mathrm{P}=1, \mathrm{~V}=\operatorname{tr}, \mathrm{O}=\operatorname{dir} .0 \mathrm{bj}, \mathrm{i}$

97. neither doe I imagin anie thing - E I.4.7.

98. then do I finde neither mercies - M III.1.26.

99. neither doe I thanke thee - LM V.4.66.

2.4.4. $\mathrm{C}=\mathrm{m}, \mathrm{T}=\mathrm{pr}, \mathrm{N}=\mathrm{sg}, \mathrm{P}=1, \mathrm{~V}=\operatorname{tr}, \mathrm{O}=$ obj.cl, $\mathrm{i}$ 100. then do I not maruell thou remembrest not - E V.1.37.

2.4.5. $\mathrm{C}=\mathrm{m}, \mathrm{T}=\mathrm{pr}, \mathrm{N}=\mathrm{sg}, \mathrm{P}=1, \mathrm{~V}=$ intr

101. I doe not so much stand - C I.3.28.

102. "in faith I do not loue". - SP III.4.27.

2.4.6. $\mathrm{C}=\mathrm{m}, \mathrm{T}=\mathrm{pr}, \mathrm{N}=\mathrm{sg}, \mathrm{P}=2, \mathrm{~V}=\mathrm{tr}, \mathrm{O}=$ dir.obj, $\operatorname{Prn}=$ rfl.prn 103. Thou doest not flatter thy selfe - SP II.1.5. 
2.4.7. $\mathrm{C}=\mathrm{m}, \mathrm{T}=\mathrm{pr}, \mathrm{N}=\mathrm{sg}, \mathrm{P}=3, \mathrm{~V}=$ intr

104. Tellus doth not speak false. - E V.3.136.

2.4.8. $\mathrm{C}=\mathrm{m}, \mathrm{T}=\mathrm{pr}, \mathrm{N}=\mathrm{pl}, \mathrm{P}=1, \mathrm{~V}=\mathrm{tr}, \mathrm{O}=\operatorname{dir} . \mathrm{obj}$ 105. We doe not meane fortune tellers $-G$ V.3.181.

2.4.9. $\mathrm{C}=\mathrm{m}, \mathrm{T}=\mathrm{pr}, \mathrm{N}=$ you1, $\mathrm{P}=2, \mathrm{~V}=$ intr 106. you do not mistrust. - C IV.4.5.

2.4.10. $\mathrm{C}=\mathrm{m}, \mathrm{T}=$ pa, $\mathrm{N}=\mathrm{sg}, \mathrm{P}=1, \mathrm{~V}=\mathrm{tr}, \mathrm{O}=$ prep.obj, $\mathrm{i}$ 107. for yet did I neuer heare of a woman - SP III.3.57.

2.4.11. $\mathrm{C}=\mathrm{m}, \mathrm{T}=$ pa, $\mathrm{N}=\mathrm{sg}, \mathrm{P}=1, \mathrm{~V}=\operatorname{tr}, \mathrm{O}=$ obj.cl, $\mathrm{i}$ 108. for yet did I neuer heare that his oracles were $-M$ V.1.37.

2.4.12. $\mathrm{C}=\mathrm{m}, \mathrm{T}=$ pa, $\mathrm{N}=\mathrm{sg}, \mathrm{P}=1, \mathrm{~V}=$ intr 109. I did not runne awaye - C I.2.27.

2.4.13. $\mathrm{C}=\mathrm{m}, \mathrm{T}=\mathrm{pa}, \mathrm{N}=\mathrm{sg}, \mathrm{P}=2, \mathrm{~V}=\mathrm{tr}, \mathrm{O}=\operatorname{dir} . \mathrm{obj}$ 110. thou didst neuer yet deceiue vertue - C I.1.29.

2.4.14. $\mathrm{C}=\mathrm{m}, \mathrm{T}=\mathrm{pa}, \mathrm{N}=\mathrm{sg}, \mathrm{P}=2, \mathrm{~V}=$ intr 111. happie Niobe...didst neuer turne - LM V.4.96.

2.4.15. $\mathrm{C}=\mathrm{m}, \mathrm{T}=\mathrm{pa}, \mathrm{N}=\mathrm{sg}, \mathrm{P}=3, \mathrm{~V}=$ intr, $\mathrm{i}$ 112. there did neuer enter any motion of loue. - LM II.1.46.

2.4.16. $\mathrm{C}=\mathrm{d}, \mathrm{T}=\mathrm{pr}, \mathrm{N}=\mathrm{sg}, \mathrm{P}=1, \mathrm{~V}=$ intr 113. I doe not dissemble - MB V.3.312.

2.4.17. $\mathrm{C}=\mathrm{d}, \mathrm{T}=\mathrm{pr}, \mathrm{N}=\mathrm{sg}, \mathrm{P}=2, \mathrm{~V}=\mathrm{tr}, \mathrm{O}=\operatorname{dir} . \mathrm{obj}, \mathrm{M}=$ cond 114. if thou doo not beleeue it - E III.4.187.

2.4.18. $C=d, T=p a, N=s g, P=2, V=\operatorname{tr}, O=$ dir.obj 115. in that thou didste not counterfeite money. - C I.3.122.

2.4.19. $\mathrm{C}=\mathrm{d}, \mathrm{T}=$ pa, $\mathrm{N}=\mathrm{sg}, \mathrm{P}=3, \mathrm{~V}=\mathrm{tr}, \mathrm{O}=\operatorname{dir} . \mathrm{obj}$ 116. because vertue neuer yet did trust fortune. $-\mathrm{C}$ I.1.30.

2.4.20. $\mathrm{C}=\mathrm{d}, \mathrm{T}=\mathrm{pa}, \mathrm{N}=\mathrm{sg}, \mathrm{P}=3, \mathrm{~V}=$ intr 117. who yet neuer did hurt. - MB III.1.28. 


\subsection{The interrogative form}

\subsubsection{Yes-no questions}

2.5.1.1. $\mathrm{C}=\mathrm{m}, \mathrm{T}=\mathrm{pr}, \mathrm{N}=\mathrm{sg}, \mathrm{P}=1, \mathrm{~V}=$ intr 118. doe I mistake? - E I.1.16.

2.5.1.2. $\mathrm{C}=\mathrm{m}, \mathrm{T}=\mathrm{pr}, \mathrm{N}=\mathrm{sg}, \mathrm{P}=2, \mathrm{~V}=\operatorname{tr}, \mathrm{O}=\operatorname{dir} . \mathrm{obj}$ 119. Doost thou see any thing? - E III.4.154.

120. Doost thou weare Cupids Quiuer - E IV.3.120.

121. dost thou think me a foole - MB I.1.22.

122. doest thou imagine thy misters - MB I.1.24.

123. Doest thou mocke me - MB II.1.24.

124. doest thou thinke them rattes - MB V.3.270.

125. Dost thou disdaine Loue - LM III.1.11.

2.5.1.3. $\mathrm{C}=\mathrm{m}, \mathrm{T}=\mathrm{pr}, \mathrm{N}=\mathrm{sg}, \mathrm{P}=2, \mathrm{~V}=\mathrm{tr}, \mathrm{O}=$ obj.cl 126. or dost thou...more delight to be hewed - C III.5.23.

127. Doest thou beleue that there are any gods - C V.1.18.

128. dost thou thinke that he will make it - G 11.3.81.

129. doest thou thinke haires will breede - M III.2.65.

130. Doest thou thinke she tooke her - MB 1.2.13.

131. doest thou thinke an horse can speake? - MB IV.2.184.

2.5.1.4. $\mathrm{C}=\mathrm{m}, \mathrm{T}=\mathrm{pr}, \mathrm{N}=\mathrm{sg}, \mathrm{P}=2, \mathrm{~V}=$ intr

132. Doest thou preferre - C IV.2.2.

2.5.1.5. $\mathrm{C}=\mathrm{m}, \mathrm{T}=\mathrm{pr}, \mathrm{N}=\mathrm{sg}, \mathrm{P}=3, \mathrm{~V}=\mathrm{tr}, \mathrm{O}=\operatorname{dir}$.obj 133. Doth Sapho bewitch thee - SP II.4.4.

134. Doth thys Monster deuoure her? - G I.1.53.

135. Doth she adde craft - G III.4.70.

136. Or dooth the remembraunce...refine my spirits - E IV.3.20.

137. Doth she want eares? - M I.2.54.

138. doth she weare any - M I.2.119.

139. Or doth Ceres... reward me - LM I.2.97.

140. Doth Erisicthon offer force - LM II.1.1.

141. doth hee stretch his hand - LM II.1.3.

2.5.1.6. $\mathrm{C}=\mathrm{m}, \mathrm{T}=\mathrm{pr}, \mathrm{N}=\mathrm{sg}, \mathrm{P}=3, \mathrm{~V}=\mathrm{tr}, \mathrm{O}=$ prep.obj

142. Doth Pan talke of the passions - M IV.1.17.

2.5.1.7. $\mathrm{C}=\mathrm{m}, \mathrm{T}=\mathrm{pr}, \mathrm{N}=\mathrm{sg}, \mathrm{P}=3, \mathrm{~V}=\operatorname{tr}, \mathrm{O}=$ obj.cl 143. doth she thinke to contend - SP IV.2.22.

144. or dooth ouercarefulnes make you forget - G I.1.73. 
145. Doth Midas determine to tempt - M I.1.59.

146. Doeth your Maiestie begin to melt - M III.1.67.

2.5.1.8. $\mathrm{C}=\mathrm{m}, \mathrm{N}=\mathrm{sg}, \mathrm{P}=3, \mathrm{~V}=\operatorname{intr}$ 147. doth it suffise? - G V.3.171.

148. Doth Dipsas stoope? - E V.2.55.

$$
\text { 2.5.1.9. } \mathrm{C}=\mathrm{m}, \mathrm{T}=\mathrm{pr}, \mathrm{N}=\mathrm{pl}, \mathrm{P}=2, \mathrm{~V}=\operatorname{tr}, \mathrm{O}=\operatorname{dir} . \mathrm{obj}
$$

149. Doe you both beeing Maidens loue one another? - G V.3.116.

150. But do you heare the strange newes - M I.2.121.

$$
\text { 2.5.1.10. } \mathrm{C}=\mathrm{m}, \mathrm{T}=\mathrm{pr}, \mathrm{N}=\mathrm{pl}, \mathrm{P}=2, \mathrm{~V}=\mathrm{tr}, \mathrm{O}=\text { obj.cl }
$$

151. Doe you thinke Ile be iested out - MB IV.2.203.

152. do you thinke Ile bee cosned - MB V.3.320.

$$
\text { 2.5.1.11. } \mathrm{C}=\mathrm{m}, \mathrm{T}=\mathrm{pr}, \mathrm{N}=\mathrm{pl}, \mathrm{P}=2, \mathrm{~V}=\text { intr }
$$

153. doe you heare? - SP III.3.128.

2.5.1.12. $\mathrm{C}=\mathrm{m}, \mathrm{T}=\mathrm{pr}, \mathrm{N}=\mathrm{pl}, \mathrm{P}=3, \mathrm{~V}=\mathrm{tr}, \mathrm{O}=$ prep.obj 154. Doe they dote so much on their daughters - G V.3.11.

$$
\text { 2.5.1.13. } \mathrm{C}=\mathrm{m}, \mathrm{T}=\mathrm{pr}, \mathrm{N}=\mathrm{pl}, \mathrm{P}=3, \mathrm{~V}=\mathrm{tr}, \mathrm{O}=\text { obj.cl }
$$

155. doe your eyes begin to loue collours - G III.4.51.

156. doe men beginne to bee equall $-\mathrm{G}$ V.3.10.

$$
\text { 2.5.1.14. } \mathrm{C}=\mathrm{m}, \mathrm{T}=\mathrm{pr}, \mathrm{N}=\mathrm{pl}, \mathrm{P}=3, \mathrm{~V}=\text { intr }
$$

157. doe your sheepe feede - G II.1.47.

158. Doe sillie Sheepeheards goe - G II.2.15.

159. doe they speake so? - G II.3.21.

160. Doe they all dissemble? E II.1.66.

161. do they still ake? - M III.2.80.

162. Doo your loues continue? - LM V.4.59.

$$
\text { 2.5.1.15. } \mathrm{C}=\mathrm{m}, \mathrm{T}=\mathrm{pr}, \mathrm{N}=\text { you } 1, \mathrm{P}=2, \mathrm{~V}=\mathrm{tr}, \mathrm{O}=\operatorname{dir} . \mathrm{obj}
$$

163. do you loue Apelles? - C V.4.121.

164. Doe you loue Campaspe? - C V.4.125.

165. do you keep the ferry - SP I.1.50.

166. Doe you knowe in court any - SP I.2.35.

167. And doe you loue him still? - SP V.2.35.

168. Do you loue the Moone Endimion? - E I.1.16.

169. doe you...continue you constant loue? - LM V.4.30.

2.5.1.16. $\mathrm{C}=\mathrm{m}, \mathrm{T}=\mathrm{pr}, \mathrm{N}=$ you1, $\mathrm{P}=2, \mathrm{~V}=\mathrm{tr}, \mathrm{O}=$ obj.cl 170. do you thinke... any use to dissemble? - SP I.2.34.

171. doe you thinke that lust followeth loue? - LM II.1.122. 
2.5.1.17. $\mathrm{C}=\mathrm{m}, \mathrm{T}=\mathrm{pr}, \mathrm{N}=$ you1, $\mathrm{P}=2, \mathrm{~V}=$ intr

172. doe you loue - SP II.1.33.

173. doe you loyter - G II.3.96.

2.5.1.18. $\mathrm{C}=\mathrm{m}, \mathrm{T}=\mathrm{pa}, \mathrm{N}=\mathrm{sg}, \mathrm{P}=2, \mathrm{~V}=\operatorname{tr}, \mathrm{O}=\operatorname{dir} . \mathrm{obj}$

174. Didst thou nourse me - SP III.3.90.

175. didst thou euer heare such a dolt? - E I.3.81.

176. diddest thou euer heare such a sighing - E II.2.1.

177. diddest thou heare anie wordes - MB V.3.154.

2.5.1.19. $\mathrm{C}=\mathrm{m}, \mathrm{T}=\mathrm{pa}, \mathrm{N}=\mathrm{sg}, \mathrm{P}=2, \mathrm{~V}=$ intr.

178. Didst thou heare so? - SP I.1.67.

2.5.1.20. $\mathrm{C}=\mathrm{m}, \mathrm{T}=\mathrm{pa}, \mathrm{N}=\mathrm{sg}, \mathrm{P}=3, \mathrm{~V}=\operatorname{tr}, \mathrm{O}=$ dir.obj

179. Did thy mother...sende thee - G III.4.69.

2.5.1.21. $\mathrm{C}=\mathrm{m}, \mathrm{T}=\mathrm{pa}, \mathrm{N}=\mathrm{pl}, \mathrm{P}=2, \mathrm{~V}=\mathrm{tr}, \mathrm{O}=$ obj.cl

180. Dyd you euer see water buble as the Sea did? - G I.4.10.

2.5.1.22. $\mathrm{C}=\mathrm{m}, \mathrm{T}=$ pa, $\mathrm{N}=$ you $1, \mathrm{P}=2, \mathrm{~V}=\operatorname{tr}, \mathrm{O}=\operatorname{dir} . \mathrm{obj}$ 181. Did you euer see any so perplexed? $-\mathrm{C}$ V.4.10.

2.5.1.23. $\mathrm{C}=\mathrm{m}, \mathrm{T}=$ pa, $\mathrm{N}=$ youl, $\mathrm{P}=2, \mathrm{~V}=\mathrm{tr}, \mathrm{O}=$ obj.cl 182. Did you euer see me kisse my Daughter? - G IV.1.47.

2.5.1.24. $\mathrm{C}=\mathrm{m}, \mathrm{T}=$ pa, $\mathrm{N}=$ you $1, \mathrm{P}=2, \mathrm{~V}=$ intr 183. did you once love? - E III.4.57.

\subsubsection{Wh-questions}

2.5.2.1. $\mathrm{C}=\mathrm{m}, \mathrm{T}=\mathrm{pr}, \mathrm{N}=\mathrm{sg}, \mathrm{P}=1, \mathrm{~V}=\operatorname{tr}, \mathrm{O}=\operatorname{dir} . \mathrm{obj}$ 184. What doe I heare? - G II.3.89.

185. Why doe I trifle the time in words? - E III.4.103.

186. What doe I see - E V.1.30.

187. whom doe I beholde - E V.1.44.

188. What doe I heare? - E V.2.86.

189. Whome doe I see? - LM V.4.38.

2.5.2.2. $\mathrm{C}=\mathrm{m}, \mathrm{T}=\mathrm{pr}, \mathrm{N}=\mathrm{sg}, \mathrm{P}=2, \mathrm{~V}=\mathrm{tr}, \mathrm{O}=\operatorname{dir} . \mathrm{obj}$

190. what dost thou thinke - C I.3.133.

191. What dost thou want? - C II.2.140.

192. What dost thou think - C V.4.45.

193. what dost thou think - C V.4.57.

194. What dost thou dislyke - C V.4.64. 
195. Whom doest thou seeke? - SP II.3.41.

196. But what doest thou answere? - SP II.3.72.

197. But why doost thou blame him - G II.1.9.

198. what doost thou feare? - G IV.4.35.

199. What doost thou feele - E V.2.18.

200. What doest thou pay - M III.2.21.

201. why doost thou abuse the world - M V.3.6.

202. what dost thou chiefest desire? - LM II.1.93.

203. What doest thou most hate - LM II.1.96.

204. What dost thou heare - LM IV.2.84.

2.5.2.3. $\mathrm{C}=\mathrm{m}, \mathrm{T}=\mathrm{pr}, \mathrm{N}=\mathrm{sg}, \mathrm{P}=2, \mathrm{~V}=\mathrm{tr}, \mathrm{O}=$ prep.obj 205. What dost thou seeke for here? - C II.1.9.

$$
\text { 2.5.2.4. } \mathrm{C}=\mathrm{m}, \mathrm{T}=\mathrm{pr}, \mathrm{N}=\mathrm{sg}, \mathrm{P}=2, \mathrm{~V}=\text { intr }
$$

206. But how doest thou then liue? - C I.2.81.

207. But why doest thou laugh. - MB II.1.84.

$$
\text { 2.5.2.5. } \mathrm{C}=\mathrm{m}, \mathrm{T}=\mathrm{pr}, \mathrm{N}=\mathrm{sg}, \mathrm{P}=3, \mathrm{~V}=\mathrm{tr}, \mathrm{O}=\operatorname{dir} . \mathrm{obj}
$$

208. whether doth the sea...bring...creatures? - C I.3.94.

209. what dishes...doth his Lordshippe feast you - E V.2.7.

210. What conditions doth she aske? - E V.2.59.

2.5.2.6. $\mathrm{C}=\mathrm{m}, \mathrm{T}=\mathrm{pr}, \mathrm{N}=\mathrm{pl}, \mathrm{P}=1, \mathrm{~V}=\mathrm{tr}, \mathrm{O}=\operatorname{dir} . \mathrm{obj}$ 211. what els do we se now then a kind of softnes - C IV.3.7.

2.5.2.7. $\mathrm{C}=\mathrm{m}, \mathrm{T}=\mathrm{pr}, \mathrm{N}=\mathrm{pl}, \mathrm{P}=2, \mathrm{~V}=\operatorname{tr}, \mathrm{O}=\operatorname{dir} . \mathrm{obj}$ 212. What doo you giue me the boots? - MB IV.2.32.

213. What punishment doe you desire - LM IV.1.80.

2.5.2.8. $\mathrm{C}=\mathrm{m}, \mathrm{T}=\mathrm{pr}, \mathrm{N}=\mathrm{pl}, \mathrm{P}=2, \mathrm{~V}=\mathrm{tr}, \mathrm{O}=$ prep.obj 214. Why do you rap so hard at the doore? - MB III.4.83.

2.5.2.9. $\mathrm{C}=\mathrm{m}, \mathrm{T}=\mathrm{pr}, \mathrm{N}=\mathrm{pl}, \mathrm{P}=3, \mathrm{~V}=\operatorname{tr}, \mathrm{O}=\operatorname{dir} . \mathrm{obj}$ 215. why doe others terme thee vnconstant - E I.1.30.

216. What do they thinke - LM IV.1.48.

2.5.2.10. $\mathrm{C}=\mathrm{m}, \mathrm{T}=\mathrm{pr}, \mathrm{N}=$ youl, $\mathrm{P}=2, \mathrm{~V}=\operatorname{tr}, \mathrm{O}=\operatorname{dir} . \mathrm{obj}$ 217. how doe yee like the sweete face - C II.2.2.

218. Whom do you loue - C IV.2.37.

219. whom do you honour - C V.2.40.

220. what do you think $-\mathrm{C}$ V.4.30.

221. what doe you think - SP III.1.37.

222. Where...doe you feele...paine? - SP III.3.8. 
223. what do you thinke - SP III.4.75.

224. what doe you call it? - G I.2.14.

225. Why doe you lay that knot - G IV.2.46.

226. Why doe you put that - G IV.2.57.

227. Why doe you couet many - LM III.1.100.

2.5.2.11. $\mathrm{C}=\mathrm{m}, \mathrm{T}=\mathrm{pr}, \mathrm{N}=$ you $1, \mathrm{P}=2, \mathrm{~V}=\operatorname{tr}, \mathrm{O}=$ dir.obj, $\mathrm{Prn}=\mathrm{rfl}$.prn 228. how doe you feele your self - SP I.2.2.

2.5.2.12. $\mathrm{C}=\mathrm{m}, \mathrm{T}=\mathrm{pr}, \mathrm{N}=$ you $1, \mathrm{P}=2, \mathrm{~V}=\mathrm{tr}, \mathrm{O}=$ obj.cl 229. But what do you imagine it to be? - C V.4.34.

2.5.2.13. $\mathrm{C}=\mathrm{m}, \mathrm{T}=\mathrm{pr}, \mathrm{N}=$ you1, $\mathrm{P}=2, \mathrm{~V}=$ intr 230. Where doe you first begin $-\mathrm{C}$ III.4.70.

231. why do you laugh? - C V.1.10.

232. why doe you sigh - SP III.4.68.

2.5.2.14. $\mathrm{C}=\mathrm{m}, \mathrm{T}=\mathrm{pa}, \mathrm{N}=\mathrm{sg}, \mathrm{P}=1, \mathrm{~V}=\mathrm{tr}, \mathrm{O}=$ obj.cl 233. why did I couet to get...crownes - M III.1.14.

234. Why did I wish that all might bee gold - M III.1.43.

2.5.2.15. $\mathrm{C}=\mathrm{m}, \mathrm{T}=\mathrm{pa}, \mathrm{N}=\mathrm{sg}, \mathrm{P}=1, \mathrm{~V}=$ intr 235. when did I rest? - E II.3.22.

236. Where did I consent - MB V.3.177.

2.5.2.16. $\mathrm{C}=\mathrm{m}, \mathrm{T}=\mathrm{pa}, \mathrm{N}=\mathrm{sg}, \mathrm{P}=2, \mathrm{~V}=\operatorname{tr}, \mathrm{O}=\operatorname{dir} . \mathrm{obj}$ 237. why didst thou draw it - C III.5.40.

238. why didst thou wound me - SP IV.1.4.

239. What diddest thou imagine - E V.1.118.

240. why didst thou boare him - MB IV.2.195.

241: Why didst thou...torment...Nymphes - LM II.1.101.

2.5.2.17. $\mathrm{C}=\mathrm{m}, \mathrm{T}=\mathrm{pa}, \mathrm{N}=\mathrm{sg}, \mathrm{P}=2, \mathrm{~V}=\operatorname{tr}, \mathrm{O}=$ obj.cl

242. But why diddest thou tell mee it was...sonne? - MB IV.2.133.

2.5.2.18. $\mathrm{C}=\mathrm{m}, \mathrm{T}=\mathrm{pa}, \mathrm{N}=\mathrm{sg}, \mathrm{P}=2, \mathrm{~V}=\operatorname{intr}$

243. why didst thou proue - SP IV.1.6.

244. How then didst thou liue? - G V.1.33.

245. how didst thou escape - LM IV.2.1.

2.5.2.19. $\mathrm{C}=\mathrm{m}, \mathrm{T}=\mathrm{pa}, \mathrm{N}=\mathrm{sg}, \mathrm{P}=3, \mathrm{~V}=\mathrm{tr}, \mathrm{O}=\operatorname{dir}$.obj

246. Why did Nature...giue a face $-\mathrm{G}$ II.4.8.

247. How did it take you - G III.1.55.

248. how did it take you - G III.1.58.

249. Why did Cupid punish vs - G IV.2.66. 
2.5.2.20. $\mathrm{C}=\mathrm{m}, \mathrm{T}=\mathrm{pa}, \mathrm{N}=\mathrm{pl}, \mathrm{P}=3, \mathrm{~V}=\mathrm{tr}, \mathrm{O}=\operatorname{dir} . \mathrm{obj}$ 250. What did they see? - E III.4.43.

2.5.2.21. $\mathrm{C}=\mathrm{m}, \mathrm{T}=$ pa, $\mathrm{N}=$ you $1, \mathrm{P}=2, \mathrm{~V}=\operatorname{tr}, \mathrm{O}=\operatorname{dir} . \mathrm{obj}$ 251. What did you dreame - SP IV.3.53.

2.6. The interrogative-negative form

2.6.1. $\mathrm{C}=\mathrm{m}, \mathrm{T}=\mathrm{pr}, \mathrm{N}=\mathrm{sg}, \mathrm{P}=2, \mathrm{~V}=\mathrm{tr}, \mathrm{O}=$ dir.obj

252. Why thẽ doest thou ow no reuerẽce $-\mathrm{C}$ II.2.125.

253. Doest thou not vnderstand their language? - M IV.3.18.

2.6.2. $\mathrm{C}=\mathrm{m}, \mathrm{T}=\mathrm{pr}, \mathrm{N}=\mathrm{sg}, \mathrm{P}=2, \mathrm{~V}=\mathrm{tr}, \mathrm{O}=$ obj.cl 254. doest thou not remember that wee haue $-\mathrm{C}$ III.2.13.

255. Doost thou not knowe that I was calculating - G III.3.34.

256. Doost thou not know what a Poet is? - E I.3.15.

257. doest thou not think she was chast? - M III.3.15.

258. Dost thou not know...that...Bacchus commaunded mee - M V.1.41.

2.6.3. $\mathrm{C}=\mathrm{m}, \mathrm{T}=\mathrm{pr}, \mathrm{N}=\mathrm{sg}, \mathrm{P}=3, \mathrm{~V}=\operatorname{tr}, \mathrm{O}=\operatorname{dir} . \mathrm{obj}$ 259. doeth not your beauty put the painter - C III.4.59.

260. What holes doth not gold bore - M I.1.62.

2.6.4. $\mathrm{C}=\mathrm{m}, \mathrm{T}=\mathrm{pr}, \mathrm{N}=\mathrm{sg}, \mathrm{P}=3, \mathrm{~V}=\mathrm{tr}, \mathrm{O}=$ obj.cl 261. dooth not that make...blushe, that makes $-\mathrm{G}$ III.4.16.

2.6.5. $\mathrm{C}=\mathrm{m}, \mathrm{T}=\mathrm{pr}, \mathrm{N}=\mathrm{sg}, \mathrm{P}=3, \mathrm{~V}=$ intr 262. doth not this matter cotton - C III.4.117.

263. Doth not the horse trauell - SP III.2.16.

264. Doth not Frankinsence \& Myrrhe breath out - E I.2.23.

265. doth not Cupid speake - LM II.1.134.

2.6.6. $\mathrm{C}=\mathrm{m}, \mathrm{T}=\mathrm{pr}, \mathrm{N}=\mathrm{pl}, \mathrm{P}=1, \mathrm{~V}=\mathrm{tr}, \mathrm{O}=$ obj.cl 266. doe we not presently say, go too - SP I.4.45.

2.6.7. $\mathrm{C}=\mathrm{m}, \mathrm{T}=\mathrm{pr}, \mathrm{N}=\mathrm{pl}, \mathrm{P}=3, \mathrm{~V}=$ intr 267. Doe not all his Subiects...swarme - M III.1.58.

2.6.8. $\mathrm{C}=\mathrm{m}, \mathrm{T}=\mathrm{pr}, \mathrm{N}=$ you $1, \mathrm{P}=2, \mathrm{~V}=\mathrm{tr}, \mathrm{O}=\operatorname{dir}$.obj 268. doe you iudge no Merchants - LM III.2.46.

2.6.9. $\mathrm{C}=\mathrm{m}, \mathrm{T}=\mathrm{pr}, \mathrm{N}=$ you $1, \mathrm{P}=2, \mathrm{~V}=\mathrm{tr}, \mathrm{O}=$ obj.cl 269. Doe you not knowe...that an honorable death is - G I.1.73. 270. do you not thinke it would beate - MB V.3.120. 
2.6.10. $\mathrm{C}=\mathrm{m}, \mathrm{T}=$ pr, $\mathrm{N}=$ you1, $\mathrm{P}=2, \mathrm{~V}=\mathrm{tr}, \mathrm{O}=$ prep.obj 271. doe you not looke on faire Ladies - SP I.2.10.

2.6.11. $\mathrm{C}=\mathrm{m}, \mathrm{T}=\mathrm{pr}, \mathrm{N}=$ you1, $\mathrm{P}=2, \mathrm{~V}=$ intr 272. doe you not bleede? - E I.3.56.

2.6.12. $\mathrm{C}=\mathrm{m}, \mathrm{T}=$ pa, $\mathrm{N}=\mathrm{sg}, \mathrm{P}=1, \mathrm{~V}=\operatorname{tr}, \mathrm{O}=\operatorname{dir} . \mathrm{obj}$ 273. dyd not I see...your daughter - G IV.1.34.

2.6.13. $\mathrm{C}=\mathrm{m}, \mathrm{T}=\mathrm{pa}, \mathrm{N}=\mathrm{sg}, \mathrm{P}=1, \mathrm{~V}=\mathrm{tr}, \mathrm{O}=$ obj.cl 274. Did not I see thee come out - C IV.1.55.

2.6.14. $\mathrm{C}=\mathrm{m}, \mathrm{T}=\mathrm{pa}, \mathrm{N}=\mathrm{sg}, \mathrm{P}=1, \mathrm{~V}=$ intr 275. when did I not sigh? - E II.3.22.

2.6.15. $\mathrm{C}=\mathrm{m}, \mathrm{T}=\mathrm{pa}, \mathrm{N}=\mathrm{sg}, \mathrm{P}=2, \mathrm{~V}=\mathrm{tr}, \mathrm{O}=\operatorname{dir} . \mathrm{obj}$ 276. Didst not thou...pul up a tree - E IV.3.12.

277. Didst not thou...pull open the yron Gates - E IV.3.14.

278. why then didst not bring a stoole - MB IV.2.166.

279. Didst thou neuer see Cupid? - LM II.1.49.

2.6.16. $\mathrm{C}=\mathrm{m}, \mathrm{T}=\mathrm{pa}, \mathrm{N}=\mathrm{sg}, \mathrm{P}=2, \mathrm{~V}=\mathrm{tr}, \mathrm{O}=$ obj.cl 280. didste thou not finde I did quip thee? - C III.2.27.

281. Dydst thou neuer heare howe Iupiter came - G II.3.90.

2.6.17. $\mathrm{C}=\mathrm{m}, \mathrm{T}=\mathrm{pa}, \mathrm{N}=\mathrm{sg}, \mathrm{P}=3, \mathrm{~V}=\mathrm{tr}, \mathrm{O}=\operatorname{dir}$.obj 282. Did no bodie name Phao - SP III.3.70.

2.6.18. $\mathrm{C}=\mathrm{m}, \mathrm{T}=\mathrm{pa}, \mathrm{N}=\mathrm{sg}, \mathrm{P}=3, \mathrm{~V}=$ intr 283. did no bodie dreame - SP IV.3.22.

2.6.19. $\mathrm{C}=\mathrm{m}, \mathrm{T}=\mathrm{pa}, \mathrm{N}=\mathrm{pl}, \mathrm{P}=3, \mathrm{~V}=\mathrm{tr}, \mathrm{O}=\operatorname{dir} . \mathrm{obj}$ 284. Didde your maisters neuer teach you - C I.2.33.

2.6.20. $\mathrm{C}=\mathrm{m}, \mathrm{T}=\mathrm{pa}, \mathrm{N}=\mathrm{pl}, \mathrm{P}=3, \mathrm{~V}=\mathrm{tr}, \mathrm{O}=$ dir.obj, Prn $=$ rfl.prn 285. But did not the two fooles...court one another? - MB IV.2.163.

2.6.21. $\mathrm{C}=\mathrm{m}, \mathrm{T}=\mathrm{pa}, \mathrm{N}=\mathrm{pl}, \mathrm{P}=3, \mathrm{~V}=$ intr 286. But did neuer any Louers come hether? - E III.4.37.

2.6.22. $\mathrm{C}=\mathrm{m}, \mathrm{T}=$ pa, $\mathrm{N}=$ you $1, \mathrm{P}=2, \mathrm{~V}=\mathrm{tr}, \mathrm{O}=$ obj.cl 287. Did not you promise mee...to make - G III.3.10. 


\section{THE ANALYSIS}

3.1. The working method can be described as follows:

3.1.1.1. First we formulate the empirically verifiable predictions that follow from the working hypothesis.

3.1.1.2. In the corpus we find all instances of $d o$-support, and analyse them.

3.1.1.3. In the same corpus we find and analyse all finite verbal forms without $d o$-support, in which $d o$-support is possible (or even obligatory after the grammaticalization of do-support).

3.1.2. Every clause is analysed as to its propositional modality (affirmative, declarative-negative, interrogative, interrogative-negative), and whether it is a main or a dependent clause. Every finite verbal form is analysed as to the following grammatical categories: tense, number, verbal person, transitivity, mood, and the presence of inversion. ${ }^{3}$ With transitive verbs it is ascertained whether their object is the direct object, or the prepositional object, or even the object clause, and also, whether the transitive verb is followed by a reflexive pronoun in lieu of an object.

3.1.3. Then the following parameters of the analysed instances are processed statistically: declarative-negative form, interrogative form (yes-no questions and wh-questions), interrogative-negative form, main clause, past tense, plural (the subject you for one person excluded), plural (the subject you for one person included), first person, second person, non-third person, third person, intransitive verb, direct object, prepositional object, object clause, prepositional object and object clause together.

\subsection{Statistical results}

3.2.1. The number of instances with do-support is 287 . This number includes two exclamatory sentences and one aposiopesis.

Hence $\mathrm{n} 1=284$.

3.2.2. The number of instances without do-support is 1328 . Hence $\mathrm{n} 2=1328$.

3.2.3. Declarative-negative form

$$
+d o=29,-d o=249 \mathrm{P}_{(\mathrm{a})}=1: 2 \%: 18.7 \%, \mathrm{Id}=3.7
$$




\subsubsection{Interrogative form}

3.2.4.1. Yes-no questions

$+d o=64,-d o=30, \mathrm{P}(\mathrm{a} 2)=22.5 \%: 2.6 \%, \mathrm{Id}=9.05$

3.2.4.2. Wh-questions

$+d o=68,-d o=151, \mathrm{P}(\mathrm{a} 3)=23.9 \%: 11.4 \%, \mathrm{Id}=5$

3.2.5. Interrogative-negative form

$+d o=36,-d o=18, \mathrm{P}(\mathrm{a} 4)=12.7 \%: 1.4 \% \mathrm{Id}=6.65$

3.2.6. Main clause (interrogative \& interr.-negative form) $\mathrm{n} 1=170, \mathrm{n} 2=199$

$+d o=170,-d o=159, \mathrm{P}(\mathrm{a} 5)=100 \%: 80 \%, \mathrm{Id}=25.4$

3.2.7. Main clause (declarative-negative form)

$$
\mathrm{n} 1=29, \mathrm{n} 2=249
$$

$+d o=24,-d o=199, \mathrm{P}(\mathrm{a} 6)=82.7 \%: 79.9 \%, \mathrm{Id}=4.8$

\subsubsection{Past tense}

$+d o=85,-d o=146, \mathrm{P}(\mathrm{a} 7)=30 \%: 11 \%, \mathrm{Id}=7.3$

3.2.9. Plural ( - you for one person)

$+d o=41,-d o=248, \mathrm{P}(\mathrm{a} 8)=14.4 \%: 18.7 \%, \mathrm{Id}=2$

3.2.10. Plural ( + you for one person)

$+d o=80,-d o=420, \mathrm{P}(\mathrm{a} 9)=28.2 \%: 31.6 \%, \mathrm{Id}=1.13$

3.2.11. First person

$$
\mathrm{n} 1=152, \mathrm{n} 2=798
$$

$+d o=62,-d o=317, \mathrm{P}(\mathrm{a} 10)=40.8 \%: 39.7 \%, \mathrm{Id}=0.25$

\subsubsection{Second person}

$$
\mathrm{n} 1=222, \mathrm{n} 2=1011
$$

$+d o=132,-d o=530, \mathrm{P}(\mathrm{a} 11)=59.4 \%: 52.4 \%, \mathrm{Id}=2$

3.2.13. Non-third person

$+d o=194,-d o=847, \mathrm{P}(\mathrm{a} 12)=68.3 \%: 63.8 \%, \mathrm{Id}=1.45$

3.2.14. Third person

$+d o=90,-d o=481, \mathrm{P}(\mathrm{a} 13)=31.7 \%: 36.2 \%, \mathrm{Id}=1.45$

3.2.15. Intransitive verb

$+d o=60,-d o=320, \mathrm{P}(\mathrm{a} 14)=21.1 \%: 24.1 \%, \mathrm{Id}=1.11$ 
3.2.16. Direct object

$\mathrm{n} 1=224, \mathrm{n} 2=1008$

$+d o=154,-d o=785, \mathrm{P}(\mathrm{a} 15)=68.7 \%: 77.9 \%, \mathrm{Id}=2.8$

3.2.17. Prepositional object

$\mathrm{n} 1=165, \mathrm{n} 2=844$

$+d o=11,-d o=59, \mathrm{P}(\mathrm{a} 16)=6.6 \%: 6.9 \%, \mathrm{Id}=0.75$

3.2.18. Object clause

$\mathrm{n} 1=213, \mathrm{n} 2=949$

$+d o=59,-d o=164, \mathrm{P}(\mathrm{a} 17)=27.7 \%: 17.2 \%, \mathrm{Id}=3.33$

3.2.19. Prepositional object and object clause together $\mathrm{n} 1=244, \mathrm{n} 2=1008$

$+d o=70,-d o=223, \mathrm{P}(\mathrm{a} 18)=31.2 \%: 22.1 \%, \mathrm{Id}=2.8$

\subsection{The analysis of statistical results}

3.3.1. There were processed all favourable events with do-support (287), and all favourable events without $d o$-support in the declarative-negative, interrogative, and interrogative-negative form (448), and $30 \% 4$ of favourable events without dosupport in the affirmative form (880).

3.3.2. Of the 287 instances with $d o$-support, 88 are in the affirmative form, 29 in the declarative-negative form, 134 in the interrogative form (66 yes-no questions and 68 wh-questions), and 36 in the interrogative-negative form.

3.3.3. The instances no. 5 and no. 173 (exclamatory sentences) and no. 132 (aposiopesis) have not been included in the statistics.

3.3.4. The opposition indicative : non-indicative mood is not discussed, since the construction with $d o$-support is rare in the non-indicative mood, hence negligible from the point of view of statistics.

3.3.5. The opposition heavy : light main verbs is not discussed, snce the frequency of their occurrence with and/or without do-support is nearly the same. In analysing Lyly's works it is impossible to prove statistically that the constructions with $d o$-support appear predominantly with heavy main verbs.

The following main verbs are used most frequently:

$+d o$ : think, love, know, hear, see, and less frequently: imagine, feel, mean, begin, find, take;

-do: have, think, love, know, do, and less frequently: hear, see, come, go, honour, take; 
Heavy main verbs (e.g. counterfeite, dishonour, disdaine, mistrust, etc.) appear only rarely in complex and/or simple constructions.

\section{THE COMPARISON BETWEEN THE PREDICTIONS AND THE RESULTS} forms.

4.1. The comparison between the declarative-negative and the remaining

Contrary to our prediction, the construction without $d o$-support is used relatively more frequently in the declarative-negative form than the construction with $d o$-support, and the difference between the two relative frequencies is statistically significant. Thus the state of affairs in the declarative-negative form vs. the remaining forms does not confirm our hypothesis.

4.2. The comparison between the interrogative and the remaining forms.

In conformity, with our prediction, the construction with do-support is used relatively more frequently in the interrogative form than the construction without do-support, and the difference between the two relative frequencies is statistically significant. Thus the state of affairs in the interrogative form vs. the remaining forms does confirm our hypothesis.

4.3. The comparison between the interrogative-negative and the remaining forms.

In conformity with our prediction, the construction with $d o$-support is used relatively more frequently in the interrogative-negative form than the construction without $d o$-support, and the difference between the two relative frequencies is statistically significant. Thus the state of affairs in the interrogative-negative form vs. the remaining forms does confirm our hypothesis.

\subsection{The comparison between the main and the dependent clauses.}

Contrary to our prediction, the construction with do-support is used relatively more frequently in the main clause than the construction without $d o$-support, and the diference between the two relative frequencies is statistically significant. Thus the state of affairs in the main clause vs. the dependent clause does not confirm our hypothesis.

\subsection{The comparison between the present tense and the past tense.}

In conformity with our prediction, the construction with do-support is used relatively more frequently in the past tense than the construction without $d o$-support, 
and the difference between the two relative frequencies is statistically significant. Thus the state of affairs in the past tense vs. the present tense does confirm our hypothesis.

4.6. The comparison between the singular and the plural.

4.6.1. Contrary to our prediction, the construction with do-support is used relatively more frequently in the singular (the subject you for one person included) than the construction without do-support, and the difference between the two relative frequencies is statistically significant.

4.6.2. Contrary to our prediction, the construction with do-support is used relatively more frequently in the singular (the subject you for one person excluded) than the construction without $d o$-support. However, the difference between the two relative frequencies is not statistically significant.

4.6.3. Thus the state of affairs in the singular vs. the plural does not confirm our hypothesis.

4.7 The comparison among the verbal persons.

4.7.1. The comparison between the first and the third verbal persons.

In conformity with our prediction, the construction with do-dupport is used relatively more frequently in the first verbal person than the construction without do-support. However, the difference between the two relative frequencies is not statistically significant. Thus the state of affairs in the first vs. the third verbal person does not confirm our hypothesis.

4.7.2. The comparison between the second and the third verbal persons.

In conformity with our prediction, the construction with do-support is used relatively more frequently in the second verbal person than the construction without do-support, and the difference between the two relative frequencies is statistically significant. Thus the state of affairs in the second vs. the third verbal person does confirm our hypothesis.

4.7.3. The comparison between the third and the non-third verbal persons.

In conformity with our prediction, the construction with do-support is used relatively more frequently in the non-third verbal persons than the construction without $d o$-support. However the difference between the two relative frequencies is not statistically significant. Thus the state of affairs in the third vs. the non-third verbal persons does not confirm our hypothesis. 


\subsection{The comparison between the transitive and the intransitive verbs.}

In conformity with our prediction, the construction without do-support is used relatively more frequently in the intransitive verbs than the construction with do-support. However the difference between the two relative frequencies is not statistically significant. Thus the state of affairs in the transitive vs. the intransitive verbs does not confirm our hypothesis.

4.9. The comparison among the transitive verbs construed with direct or prepositional object, or with object clause.

4.9.1. The comparison between the verbs construed with direct object and the verbs construed with prepositional object.

Contrary to our prediction, the construction without do-support is used relatively more frequently with the verbs construed with prepositional object than the construction with $d o$-support. The difference between the two relative frequencies is statistically significant. Thus the state of affairs in the verbs construed with direct object vs. the verbs construed with prepositional object does not confirm our hypothesis.

4.9.2. The comparison between the verbs construed with direct object and the verbs construed with object clause.

In conformity with our prediction, the construction with do-support is used relatively more frequently with the verbs construed with the object clause than the construction without do-support, and the difference between the two relative frequencies is statistically significant. Thus the state of affairs in the verbs construed with direct object vs. the verbs construed with object clause does confirm our hypothesis.

4.9.3. The comparison between the verbs construed with direct object on the one side, and the verbs construed with prepositional object or object clause on the other side.

In conformity with our prediction, the construction with $d o$-support is used relatively more frequently with the verbs construed with prepositional object or object clause than the construction without do-support, and the difference between the two relative frequencies is statistically significant. Thus the state of affairs in the verbs construed with direct object vs. the verbs construed with prepositional object or object clause does confirm our hypothesis.

4.10. The analysis of our statistical results shows that our working hypothesis is confirmed with respect to the following parameters: interrogative form (yes-no 
questions, wh-questions), interrogative-negative form, past tense, second person, object clause, prepositional object and object clause together. The remaining parameters do not confirm our hypothesis, either because they disconfirm it, or because they are statistically insignificant.

4.11. Our hypothesis is fully disconfirmed by the declarative-negative form, and, conditionally, by the plural.

\section{FOOTNOTES}

1 Abbreviations:

$\mathrm{C}=$ clause, $\mathrm{m}=$ main, $\mathrm{d}=$ dependent

$\mathrm{T}=$ tense, $\mathrm{pr}=$ present, $\mathrm{pa}=$ past

$\mathbf{N}=$ number, $\mathrm{sg}=$ singular, $\mathrm{pl}=$ plural, you 1 = you for one person

$\mathbf{P}=$ person, $1=$ first, $2=$ second, $3=$ third

$\mathrm{V}=$ verb(main), $\mathrm{tr}=$ transitive, intr $=$ intransitive

$\mathrm{O}=$ object, dir.obj $=$ direct object, prep.obj $=$ prepositional object, obj.cl $=$ object clause

$\operatorname{Prn}=$ pronoun, rfl.prn $=$ reflexive pronoun

$\mathrm{M}=$ mood, cond $=$ conditional

$\mathrm{i}=$ inversion.

$2 \mathrm{E}=$ abbreviation of the title, $\mathrm{V} .=$ act, $1 .=$ scene, $54 .=$ line.

3 The inversion is more frequently used with intransitive verbs (Ellegård 1953: 190).

4 First $30 \%$ of each of the 7 plays in prose.

\section{REFERENCES}

Bond, R. W. (ed.), The Complete Works of John Lyly. 3 vols. Clarendon Press, Oxford, 1902.

Ellegård, A., The Auxiliary Do. The Establishment and Regulation of Its Use in English. Almqvist \& Wiksell, Stockholm, 1953.

\section{Povzetek}

\section{RAZVOJ SKLADENJSKE SPREMEMBE - ANGLEŠKI GLAGOL DO}

Opazovali smo skladenjsko spremembo in njen potek pri angleškem pomožnem glagolu do v obdobju (konec 16. stoletja), ko je bila zgradba z do še skladenjska inačica zgradbe brez do in kot opisna zgradba okrepitev glede na ustrezno zgradbo brez do.

Pri tem smo ugotavljali, ali okoliščine ustrezajo domnevi, po kateri naj bi se v jeziku uveljavljale okrepitve. Kot dokazno gradivo smo obdelali sedem dram v prozi iz "The Complete Works of John Lyly", Oxford 1902.

Zgradbe z do se v navedenem jezikovnem gradivu, v primerjavi z zgradbami brez do, statistično signifikantno pogosteje pojavljajo $\mathrm{v}$ vprašalni obliki (odločevalnih in vsebinskih vprašanjih), vprašalno-nikalni obliki ter v pretekliku, drugi osebi in v zgradbah s stavčnim ali polstavčnim predmetom, če jih štejemo skupaj. Vse te oblike in kategorije spadajo med zapletenejše in pogostnost pojavljanja zgradb z 
do v njih potrjuje našo domnevo o nastajanju in razvoju okrepitev v bolj zapletenem, "težkem" okolju. -Naše domneve ne potrjujeta nikalna oblika in, pogojno, množina. Ostali parametri so nesignifikantni (npr. prva oseba) in/ali pa za njih nismo ničsar napovedali (npr. neprehodni glagoli).

Iz navedenega vidimo, da se je zgradba $z$ do pojavljala $v$ zapletenem okolju pogosteje kot $v$ manj zapletenem; to pa je v skladu $\mathrm{z}$ okrepitvami, ki predstavljajo začetek skladenjskih sprememb. 